\title{
A placebo-controlled investigation of the analgesic effects, abuse liability, safety and tolerability of a range of oral cannabidiol doses in healthy humans
}

\author{
Caroline A. Arout, Margaret Haney, Evan S. Herrmann, Gillinder Bedi, Ziva D. Cooper
}

Br J Clin Pharmacol 2021; 1-9

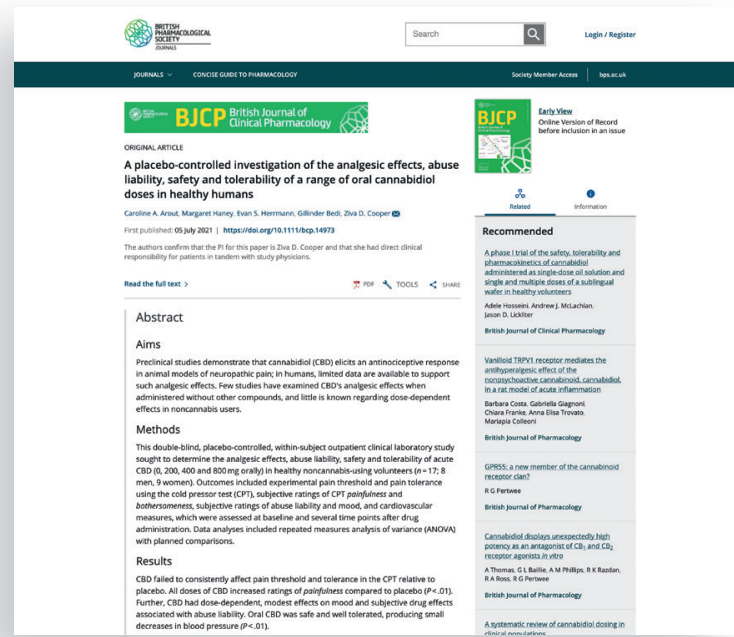

A

ufgrund der nicht toxischen Eigenschaften von Cannabidiol (CBD), seiner sehr geringen Neigung zu missbräuchlicher Verwendung und fehlender Effekte auf kognitive Fähigkeiten steht die Substanz sehr im Fokus einer möglichen Anwendung in der Schmerztherapie. Präklinische Untersuchungen an Labortieren konnten teilweise analgetische Wirkungen nachweisen, die auf Multi-Rezeptoreffekte des CBD zurückgeführt werden konnten. Die wenigen kontrollierten klinischen Studien bisher brachten nur ungenügende Hinweise auf einen robusten analgetischen Effekt. Viele Befunde, insbesondere zur Anwendung von CBD bei chronischen Schmerzsyndromen, wurden ebenfalls nur als Fallbeschreibungen publik gemacht. Deshalb beschloss eine internationale Forschergruppe am New York State Psychiatric Institute, die akuten, dosisabhängigen Effekte von CBD in Bezug auf Analgesie in einer Placebo-kontrollierten Studie an einem standardisierten akuten Schmerzreiz, dem Cold Pressure Test (= Eintauchen der Hand in $4{ }^{\circ} \mathrm{C}$ kaltes Wasser), zu untersuchen.

17 Proband*innen durchliefen randomisiert die vier Testphasen mit entweder Placebo, 200 mg, 400 mg oder 800 mg [+]-CBD Isomer, oral als Lösung eingenommen. Die Cold-Pressure-TestParameter Schmerzschwelle und Schmerztoleranz sowie die subjektiven Parameter allgemeines Schmerzempfinden und störendes Schmerzerlebnis wurden über sechs Stunden nach Substanz/ Placebo-Einnahme im 30-minütigen Intervall geprüft, dem möglichen therapeutischen Blutspiegelverlauf der Substanz angepasst. Zusätzlich wurden Blutdruck und Herzfrequenz bestimmt. Alle Werte wurden als Veränderung gegenüber den basalen Befunden, unmittelbar vor Substanz/Placebo-Einnahme erhoben, dargestellt.

Die Ergebnisse waren etwas überraschend, denn nur die 200-mgDosis von CBD konnte die Schmerzschwelle erhöhen, während die hohe Dosis die Schmerztoleranz sogar erniedrigte; die mittlere Dosis war ohne signifikanten Effekt. Auch die subjektiven Parameter allgemeines Schmerzempfinden und störendes Schmerzerlebnis blieben von allen CBD-Dosen unbeeinflusst. CBD erniedrigte den

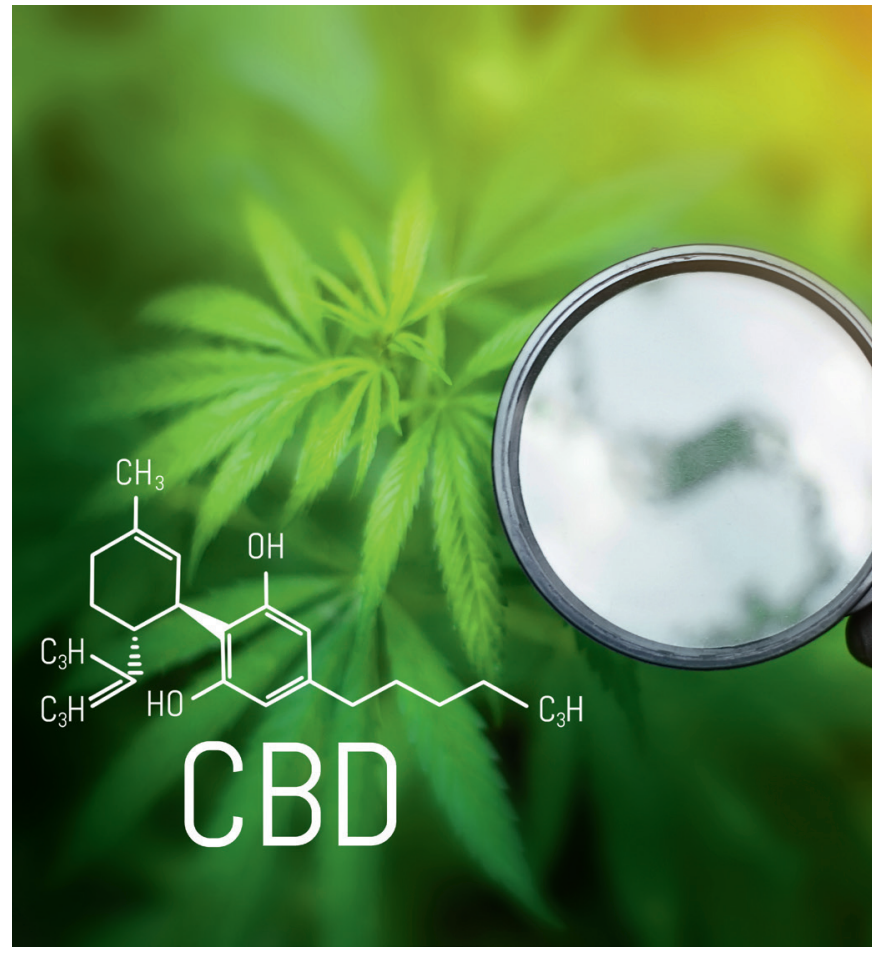

Blutdruck ohne Änderung der Herzfrequenz; berichtete Nebeneffekte nach der CBD-Applikation waren mild und klinisch unbedeutend.

In Summe muss aufgrund dieser Daten die gute Verträglichkeit von CBD, gleichzeitig aber die global fehlende akute analgetische Wirkung beim Menschen anerkannt werden. Dieses Ergebnis aus einer „randomisierten Placebo-kontrollierten Humanstudie“ ist ganz klar sehr hoch zu bewerten. Es schließt natürlich nicht aus, dass CBD bei längerfristiger Einnahme bei chronischen (entzündlichen oder neuropathischen) Schmerzsyndromen doch analgetisch wirken könnte, eine Annahme, die aber noch durch zukünftige Studien mit ausreichenden Messparametern und Kontrollen zu beweisen sein wird.

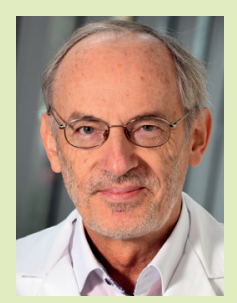

\section{KOMMENTAR}

Von Univ.-Prof. Dr. Josef Donnerer Johannes Kepler Universität, Medizinische Fakultät, Institut für Pharmakologie, Linz

Humanstudien gehen vor präklinischen Untersuchungen; prospektive randomisierte, Placebo-kontrollierte Studien gehen vor retrospektiven Fallbeschreibungen und Beobachtungsstudien. In diesem Sinne wäre die „analgetische Wirkung“ von CBD einzustufen. 